\title{
A Combinatorial Derivation of the PASEP Stationary State
}

\author{
Richard Brak ${ }^{\dagger}$, Sylvie Corteel ${ }^{b}$, John Essam ${ }^{\ddagger}$ \\ Robert Parviainen ${ }^{\dagger}$ and Andrew Rechnitzer ${ }^{\dagger}$
}

${ }^{\dagger}$ Department of Mathematics and Statistics,

The University of Melbourne,

Parkville, Victoria 3010, Australia

\author{
${ }^{b}$ Laboratoire LRI \\ Bâtiment 490 - Bureau 254 \\ Université Paris XI \\ 91405 Orsay Cedex cedex, France
}

${ }^{\ddagger}$ Department of Mathematics and Statistics,

Royal Holloway College, University of London,

Egham, Surrey TW20 0EX, England.

Submitted: Jul 17, 2006; Accepted: Nov 13, 2006; Published: Nov 23, 2006

Mathematics Subject Classifications: 05A99, 60G10

\begin{abstract}
We give a combinatorial derivation and interpretation of the weights associated with the stationary distribution of the partially asymmetric exclusion process. We define a set of weight equations, which the stationary distribution satisfies. These allow us to find explicit expressions for the stationary distribution and normalisation using both recurrences and path models. To show that the stationary distribution satisfies the weight equations, we construct a Markov chain on a larger set of generalised configurations. A bijection on this new set of configurations allows us to find the stationary distribution of the new chain. We then show that a subset of the generalised configurations is equivalent to the original process and that the stationary distribution on this subset is simply related to that of the original chain. We also provide a direct proof of the validity of the weight equations.
\end{abstract}




\section{Introduction}

The PASEP (Partially asymmetric exclusion process) is a generalisation of the TASEP. This model was introduced by physicists $[2,8,9,10,11,16]$. The TASEP consists of (black) particles entering a row of $n$ cells, each of which is occupied by a particle or vacant. A particle may enter the system from the left hand side, hop to the right and leave the system from the right hand side, with the constraint that a cell contains at most one particle. The particles in the PASEP move in the same way as those in the TASEP, but in addition may enter the system from the right hand side, hop to the left and leave the system from the left hand side, as illustrated in Figure 1.

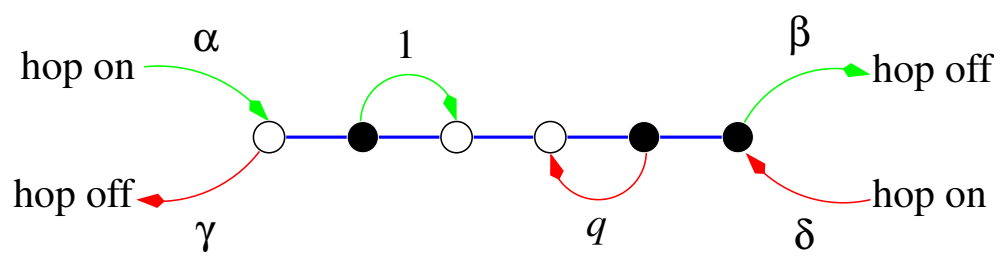

Figure 1: The PASEP.

From now on we will say that the empty cells are filled with white particles. A basic configuration is a row of $n$ cells, each containing either a black, $\bullet$, or a white, o, particle. Let $\mathcal{B}_{n}$ be the set of basic configurations of $n$ particles. We write these configurations as words of length $n$ in the language $\{\circ, \bullet\}^{*}$, so that $\bullet^{k}$ denotes a string of $k$ black particles and $A B$ denotes the configuration made up of the word $A$ followed by the word $B$. We denote the length of the word $A$ by $|A|$.

The PASEP is a Markov process on the set $\mathcal{B}_{n}$, with parameters $\alpha, \beta, \gamma, \delta, \eta$ and $q$, and transition intensities $g_{X, Y}$ given by

$$
\begin{aligned}
& g_{\circ B, \bullet B}=\alpha, \quad g_{A \bullet, A \circ}=\beta, \quad g_{A \bullet B, A \bullet \bullet}=\eta, \\
& g_{\bullet B, \circ B}=\gamma, \quad g_{A \circ, A \bullet}=\delta, \quad g_{A \circ \bullet B, A \bullet B}=q,
\end{aligned}
$$

and $g_{X, Y}=0$ in all other cases. It is common practise to, without loss of generality, set $\eta=1$. Unless otherwise indicated, we follow this practise in this paper.

See an example of the state space and transitions for $n=2$ in Figure 2.

There are many results for the PASEP. A central question is the computation of the stationary distribution. This has been most successfully analysed using a matrix product Ansatz $[10,16]$.

In this paper we give a combinatorial derivation and interpretation of the stationary distribution of the PASEP which is independent of the matrix product Ansatz. To our knowledge the only previous purely combinatorial derivations are for the special case of the TASEP, for example [8] together with [15] and [12, 13, 14].

Our derivation works by 


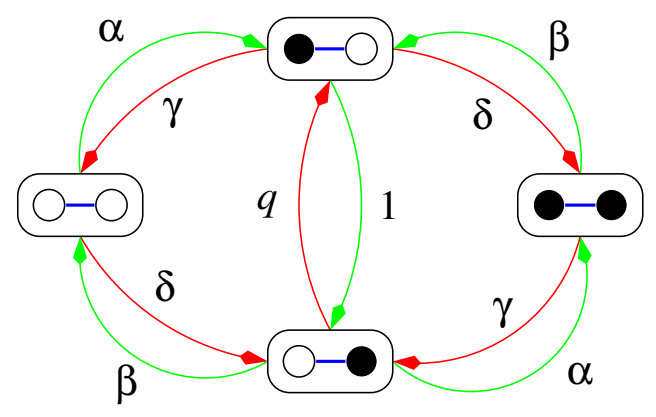

Figure 2: The transitions for $n=2$.

[i] Constructing a larger Markov chain on both basic configurations and marked basic configurations which we call marked configurations.

[ii] Using a bijection between marked configurations to find the stationary distribution of the larger chain.

[iii] Showing that a subset of the configurations is equivalent to the original chain and that the stationary state on this subset is simply related to that of the original chain.

We note that this is similar to the work done in $[12,13,14]$ where the authors studied the case $\delta=\gamma=q=0$. Here we study the stationary distribution of the full model.

We first define a set of weight equations.

Definition 1 (Basic Weight Equations). Let $W(X)$ be a real valued function defined on $\cup_{n} \mathcal{B}_{n}$. If $W(X)$ satisfies the set of equations

$$
\begin{aligned}
W(X) & =1 \text { if } X \in \mathcal{B}_{0} \\
W(X) & =\alpha W(\circ X)-\gamma W(\bullet X) \\
W(X) & =\beta W(X \bullet)-\delta W(X \circ) \\
W(A \circ B)+W(A \bullet B) & =\eta W(A \bullet \circ B)-q W(A \circ \bullet B)
\end{aligned}
$$

we call $W(X)$ a basic weight.

Below we address the issues of existence and uniqueness of solutions to the above equations by finding explicit examples of basic weights for certain values of the parameters of the model.

The main result of this paper is to give a combinatorial derivation of the following theorem:

Theorem 1. Given a basic weight $W(X)$, the stationary distribution $\mathrm{P}_{\infty}(X)$ is given by

$$
\mathrm{P}_{\infty}(X)=\frac{W(X)}{Z_{n}}
$$


where the normalisation $Z_{n}$ is

$$
Z_{n}=\sum_{X \in \mathcal{B}_{n}} W(X)
$$

We may use the basic weight equations to find expressions for $Z_{n}$. Unfortunately we have not yet found a combinatorial derivation of Sasamoto's full five parameter integral expression for $Z_{n}$, [16] (one of the six parameters can be set to one without loss of generality), but we are able to find many different specialisations. Theorem 1 is a generalisation to arbitrary $q$ of the $q=0$ result of [8].

We also find simple expressions for the stationary distribution of certain or all configurations for particular parameter combinations. For example:

Proposition 2. If $q=1-\frac{(\alpha+\beta+\gamma+\delta)(\alpha \beta-\gamma \delta)}{(\alpha+\delta)(\beta+\gamma)}$ then

$$
\mathrm{P}_{\infty}(X)=\frac{(\beta+\gamma)^{w}(\alpha+\delta)^{n-w}}{(\alpha+\beta+\gamma+\delta)^{n}}
$$

where $w$ is the number of white particles in $X$. In particular, the number of white particles at stationarity is Binomially distributed, with parameters $n$ and $(\beta+\gamma) /(\alpha+\beta+\gamma+\delta)$.

Proposition 3. If $\gamma=\delta=0$ and $q=\alpha=\beta=1$ then

$$
\mathrm{P}_{\infty}\left(X=\bullet^{k} A\right)=\frac{(n-k+1)^{k}(n-k+1) !}{(n+1) !},
$$

where $A$ is any configuration in $\mathcal{B}_{n-k}$.

Proposition 4. If $\gamma=\delta=q=0$ and $\alpha=\beta=1 / 2$ then

$$
\mathrm{P}_{\infty}(X)=\frac{1}{2^{n}}
$$

independent of the configuration $X$.

We also prove the following proposition, first derived in [16] (via the matrix Ansatz and Askey-Wilson q-polynomials).

Proposition 5. If $\eta=q=1$, then

$$
Z_{n}=\frac{1}{(\alpha \beta-\gamma \delta)^{n}} \prod_{i=0}^{n-1}(\alpha+\beta+\gamma+\delta+i(\alpha+\gamma)(\beta+\delta))
$$

For the special case $\alpha=\beta=\gamma=\delta$ and $q=0$ we have the following conjecture for the normalisation. 
Conjecture 6. Assume $\alpha=\beta=\gamma=\delta$ and $q=0$. To avoid a denominator $\alpha \beta-\gamma \delta=0$, we rescale the weights for configurations of length 1: $W(\circ)=\beta+\gamma=2 \alpha$ and $W(\bullet)=\alpha+\delta=2 \alpha$ (previously $W(\circ)=(\beta+\gamma) /(\alpha \beta-\gamma \delta)$ and $W(\bullet)=(\alpha+\delta) /(\alpha \beta-\gamma \delta))$.

Define an auxiliary function $Q_{i, j}$ by

$$
Q_{i, j}=\frac{4^{i} j(j+(2 i-3)) ! !}{(2(i-1)) ! !(j-1) ! !} .
$$

Then the rescaled normalisation $Z_{n}^{\prime}$ is given by a polynomial in $\alpha$ with coefficients given by the anti-diagonal terms in $Q_{i, j}$. Namely,

$$
\begin{aligned}
& Z_{1}^{\prime}=\alpha^{1} Q_{1,1}=4 \alpha, \\
& Z_{2}^{\prime}=\alpha^{0}\left(Q_{1,2}+\alpha Q_{2,1}\right)=8+16 \alpha, \\
& Z_{3}^{\prime}=\alpha^{-1}\left(Q_{1,3}+\alpha Q_{2,2}+\alpha^{2} Q_{3,1}\right)=\alpha^{-1}\left(12+48 \alpha+64 \alpha^{2}\right), \\
& Z_{4}^{\prime}=\alpha^{-2}\left(Q_{1,4}+\alpha Q_{2,3}+\alpha^{2} Q_{3,2}+\alpha^{3} Q_{4,1}\right)=\alpha^{-2}\left(16+96 \alpha+240 \alpha^{2}+256 \alpha^{3}\right),
\end{aligned}
$$

and for general $n$,

$$
Z_{n}^{\prime}=\alpha^{2-n} \sum_{k=1}^{n} \alpha^{k-1} Q_{k, n+1-k}=4 \alpha^{2-n} \sum_{k=0}^{n-1} \frac{(4 \alpha)^{k}(n-k)((n+k-1) / 2) ! !}{k ! !((n-k-1) / 2) ! !} .
$$

In section 2 we use the basic weight equations to find recurrences for the normalisations and in section 3 we describe path model interpretations of the stationary states and normalisations. In section 4 proofs of the propositions and theorems stated in the earlier sections are given. In particular a first proof of theorem 1 is given in section 4.1. In section 5 we define a larger Markov chain, the M-PASEP, whose stationary distribution is related to that of the PASEP chain in section 6. The stationary distribution of the M-PASEP is given by proposition 27 which is proved in section 6 and provides a second proof of theorem 1.

\section{Recursions}

In this section we study the normalisation using the weight equations (under the assumption $\eta=1$ ). By considering the position of first (leftmost) $\circ$ particle the weight equations may be used to obtain a recursion to compute $Z_{n}$ when either $\gamma$ or $\delta$ are zero. Here we consider $\delta=0$, and note that similar results may be obtained for $\gamma=0$.

\subsection{Recursions for $Z_{n}$}

Let $W_{n, k}$ be the sum of the weights of configurations in $\mathcal{B}_{n}$ that start with exactly $k \bullet$ s and then at least one $\circ$ or are all black. Similarly let $W_{n, k, j}$ the sum of the weights of basic configurations in $\mathcal{B}_{n}$ that start with exactly $k \bullet$ s then a single $\circ$ and then at least $j \bullet$ s. Finally, let $Z_{n, k}$ be the sum of the weights of basic configurations in $\mathcal{B}_{n}$ that start with at least $k \bullet$ s. 
Proposition 7. If $\delta=0$ then $Z_{n, k}, W_{n, k}$ and $W_{n, k, j}$ satisfy the following equations

$$
\begin{aligned}
& W_{n, k}= \begin{cases}\left(Z_{n-1,0}+\gamma Z_{n, 1}\right) / \alpha & \text { if } k=0 \\
W_{n-1, k-1}+Z_{n-1, k}+q W_{n, k-1,1} & \text { if } k \in[1, n-1] \\
W_{n-1, n-1} / \beta & \text { if } k=n \\
0 & \text { if } k>n\end{cases} \\
& W_{n, k, j}= \begin{cases}W_{n, k} & \text { if } j=0 \\
\left(Z_{n-1, j}+\gamma Z_{n, j+1}\right) / \alpha & \text { if } k=0 \\
0 & \text { if } k+j>n \\
Z_{n-1, k+j}+W_{n-1, k-1, j}+q W_{n, k-1, j+1} & \text { otherwise }\end{cases} \\
& Z_{n, k}= \begin{cases}W_{n, k}+Z_{n, k+1} & \text { if } k \in[0, n-1] \\
W_{n, n} & \text { if } k=n \\
0 & \text { if } k>n\end{cases}
\end{aligned}
$$

These follow from application of the relations of the weight equations to basic configurations where the first $\bullet$ pair occurs at position $k$ for $k \in[1, n-1]$. The case $k=n$ corresponds to an all black particle configuration. Similar recurrence relations to (12) were obtained in [8] for the special case of $q=0$. In the notation of [8] $Z_{n, k}=Y_{n}(n-k+1)$.

Using these recurrences we can compute $Z_{n}=Z_{n, 0}$ for any $n$. Using this data, we were able to guess the form of $Z_{n}$ for specific $\alpha, \beta, \gamma, q$ and once guessed a simple substitution back into the recurrences (and a check of the initial boundary equations) gives the following corollaries. (The $Z_{n}$ result for $\delta=q=0, \alpha=\beta=1$ has previously appeared in $[8,10,4,12]$. In [8] the length generating function for $Z_{n, k}$ was also obtained and later $Z_{n, k}$ for arbitrary $\alpha$ and $\beta$ was found in [15].)

Corollary 8. If $\gamma=\delta=q=0$ and $\alpha=\beta=1 / 2$, then

$$
Z_{n}=2^{2 n}, \quad Z_{n, k}=2^{2 n-k}
$$

Corollary 9. If $\gamma=\delta=q=0$ and $\alpha=\beta=1$, then

$$
Z_{n}=\frac{1}{n+2}\left(\begin{array}{c}
2 n+2 \\
n+1
\end{array}\right), \quad Z_{n, k}=\frac{k+2}{n+2}\left(\begin{array}{c}
2 n-k+1 \\
n-k
\end{array}\right) .
$$

Note, $Z_{n}$ is the Catalan number $C_{n+1}$ and $Z_{n, k}$ is the Ballot number $B_{2 n+1-k, k+1}$.

Corollary 10. If $\gamma=\delta=q=0, \alpha=1$ and $\beta=1 / 2$, then

$$
Z_{n}=\left(\begin{array}{c}
2 n+1 \\
n
\end{array}\right), \quad Z_{n, k}=\left(\begin{array}{c}
2 n-k+1 \\
n-k
\end{array}\right) .
$$

Corollary 11. If $\gamma=\delta=0$ and $q=1$, then

$$
Z_{n}=\prod_{i=1}^{n}\left(\frac{1}{\alpha}+\frac{1}{\beta}+i-1\right), \quad Z_{n, k}=\left(n-k+\frac{1}{\beta}\right)^{k} Z_{n-k} .
$$


Corollary 12. If $\delta=0$ and $\gamma=q=1$, then

$$
Z_{n}=\prod_{i=1}^{n}\left(\left(\frac{1}{\alpha}+1\right)\left(\frac{1}{\beta}+i\right)-1\right), \quad Z_{n, k}=\left(n-k+\frac{1}{\beta}\right)^{k} Z_{n-k} .
$$

\section{Path models}

Another way to compute $Z_{n}$ is to give a bijection between basic configurations counted by their weights and a family of weighted lattice paths. A similar result was given in [4] for $\gamma=\delta=q=0$. In [12] "complete" configurations (these are pairs of basic configurations with additional constraints) can also be interpreted as paths when $\gamma=\delta=q=0$. Here we generalise one of the approaches of [4] to get the result for $\gamma=\delta=0$ and $q>0$, and give a similar representation for $\eta=q$ and general $\alpha, \beta, \gamma$ and $\delta$.

Definition 2. A Motzkin path, [1], of length $n$ is a sequence of vertices $p=\left(v_{0}, v_{1}, \ldots, v_{n}\right)$, with $v_{i} \in \mathbb{N}^{2}$ (where $\mathbb{N}=\{0,1, \ldots\}$ ), with steps $v_{i+1}-v_{i} \in\{(1,1),(1,-1),(1,0)\}$ and $v_{0}=(0,0)$ and $v_{n}=(n, 0)$. A bicoloured Motzkin path is a Motzkin path in which each east step is labelled by one of two colours, and generalised bicoloured Motzkin path is a Motzkin path in which all steps are labelled by one of two colours.

These paths can be mapped to words in the language $\{\stackrel{\circ}{N}, \stackrel{\bullet}{N}, \stackrel{\circ}{S}, \stackrel{\bullet}{S}, \stackrel{\circ}{E}, \dot{E}\}$ by mapping the different coloured steps $(1,1)$ to $\stackrel{\circ}{N}$ and $\stackrel{\circ}{N}$, steps $(1,-1)$ to $\stackrel{\circ}{S}$ and $\dot{S}$, and horizontal steps to $\stackrel{\circ}{E}$ and $\stackrel{\bullet}{E}$. The height of a step $v_{i+1}-v_{i}$ is the $y$-coordinate of the vertex $v_{i}$. The heights and colours of the steps determine the weights of the paths.

\subsection{Path models for $\gamma=\delta=0$.}

In this case we will only need colours on the horizontal steps. Therefore we let $\stackrel{\bullet}{N}=\stackrel{\circ}{N}=N$ and $\dot{S}=\stackrel{\circ}{S}=S$, so the language is restricted to $\{N, S, \stackrel{\circ}{E}, \stackrel{\bullet}{E}\}$.

Definition 3. Let $\mathcal{P}_{n}$ be the set of bicoloured Motzkin paths of length $n$. The weight of the path in $\mathcal{P}_{0}$ is 1 and the weight of any other path is the product of the weights of its steps. The weight $w\left(p_{k}\right)$ of a step $p_{k}$ starting at height $h$ is given by:

$$
(1-q) w\left(p_{k}\right)= \begin{cases}1-q^{h+1} & \text { if } p_{k}=N \\ 1+u q^{h} & \text { if } p_{k}=\stackrel{\circ}{E} \\ 1+v q^{h} & \text { if } p_{k}=\dot{\bullet} \\ 1-u v q^{h-1} & \text { if } p_{k}=S\end{cases}
$$

where $u=\frac{1}{\alpha}(1-q)-1, v=\frac{1}{\beta}(1-q)-1$. 


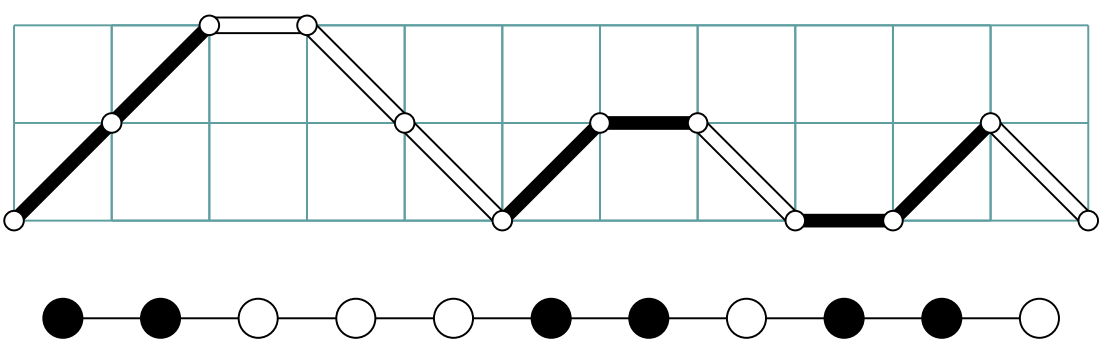

Figure 3: A path $p \in \mathcal{P}_{11}$ (top) corresponding to the word $N N \stackrel{\circ}{E} S S N \dot{E} S \dot{E} N S$, and the image configuration $\theta(p)$ (bottom).

An example of a path in $\mathcal{P}_{11}$ is given in Figure 3.

Define a mapping $\theta: \mathcal{P}_{n} \mapsto \mathcal{B}_{n}$ where each bicoloured Motzkin path is mapped to a basic configuration such that each step $S$ or $\stackrel{\circ}{E}$ is mapped to a white particle and each step $N$ or $\dot{E}$ is mapped to a black particle. This mapping is many-to-one, and we let $\theta^{-1}(X)$ denote the set of all paths that map to $X$.

Theorem 13. When $\gamma=\delta=0$ the weight of a basic configuration $X$ is given by

$$
W(X)=\sum_{p \in \theta^{-1}(X)} w(p)
$$

and

$$
Z_{n}=\sum_{p \in \mathcal{P}_{n}} w(p)
$$

This theorem gives a combinatorial derivation of the stationary distribution that does not make use of the matrix product Ansatz which was used to obtain the results in $[10,16]$. The proof is given in Section 4.4 and works by showing that the the weight of the paths obeys the same equations as the weight of the basic configurations.

We may specialise the above result to get the corollary:

Corollary 14. If $\alpha=\beta=1$ the paths $\mathcal{P}_{n}$ correspond to (uncoloured) Motzkin Paths of length $n$ where the weight of a step starting at height $h$ is $1+q+\ldots+q^{h}$.

* If $q=0$ then $Z_{n}=C_{n+1}$, where $C_{n}$ is the $n^{\text {th }}$ Catalan number.

* If $q=1$ then $Z_{n}=(n+1)$ ! (see [3, 17]).

This can be linked to well-known results on the $q$-enumeration of permutations, $[6,7]$. Also if $\alpha=\beta=1 / 2$ and $q=0$, only the paths that are made of east steps have non zero weight. Each such path has weight $2^{n}$. Therefore $W(X)=2^{n}$ for any $X \in \mathcal{B}_{n}$ and $Z_{n}=4^{n}$ in that case - this is Proposition 4.

Applying Theorem 1 of [1], we instantly get a generating function for weights. Namely, 
Corollary 15. Let $f_{w, n}$ be the sum of weights of configurations of length $n$, with exactly $w$ white particles. Further, define $F(t, z)=\sum_{w, n} f_{w, n} t^{w} z^{n}$, and let $\kappa_{h}=z\left(w \stackrel{\circ}{E}_{h}+\dot{E}_{h}\right)$ and $\lambda_{h}=z^{2} w N_{h} S_{h}$. Then

$$
F(t, z)=\frac{1}{1-\kappa_{0}-\frac{\lambda_{0}}{1-\kappa_{1}-\frac{\lambda_{1}}{1-\kappa_{2}-\cdots}}} .
$$

\subsection{Path models for $q=1$}

Definition 4. Let $\mathcal{M}_{n}$ be the set of generalised bicoloured Motzkin paths of length n. Given a basic configuration $X$, let $\mathcal{M}_{n}(X)$ be the set of generalised bicoloured Motzkin paths of length $n$ in which step $i$ have the same colour as the particle at position $i$ in $X$ (see Figure 4).

The weight of the path in $\mathcal{M}_{0}(X)$ is 1 and the weight of any other path is the product of the weights of its steps.

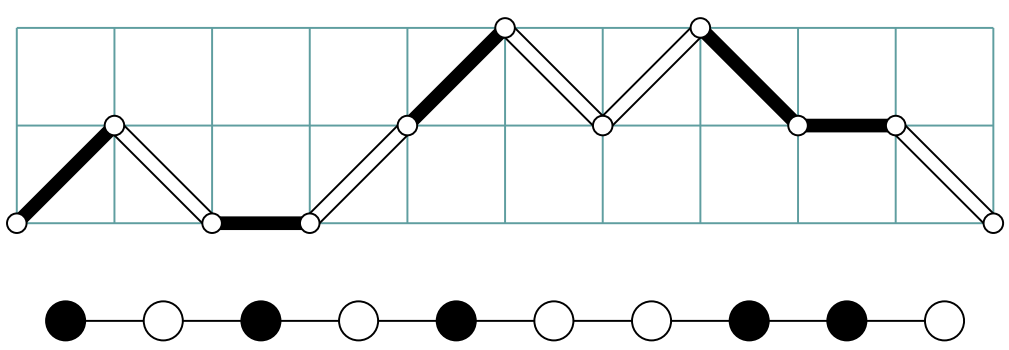

Figure 4: A generalised bicoloured Motzkin path of length 10 (top), with weight $\stackrel{\circ}{N}_{1} \stackrel{\circ}{S}_{0} \dot{E}_{0} \stackrel{\circ}{N}_{1} \stackrel{\bullet}{N}_{2} \stackrel{\circ}{S}_{1} \stackrel{\circ}{N}_{2} \stackrel{\bullet}{S}_{1} \dot{\bullet}_{1} \stackrel{\circ}{S}_{0}$, and the corresponding basic configuration (bottom).

Theorem 16. If $q=1$ there exist weights $w\left(p_{k}\right)$ such that the weight of a basic configuration $X$ is given by

$$
W(X)=\frac{1}{(\alpha \beta-\gamma \delta)^{n}} \sum_{p \in \mathcal{M}_{n}(X)} w(p),
$$

and

$$
Z_{n}=\frac{1}{(\alpha \beta-\gamma \delta)^{n}} \sum_{p \in \mathcal{M}_{n}} w(p)
$$


If $q=1$ and the weight of a step $p_{k}$ starting at height $h$ is given by:

$$
\begin{array}{lll}
\text { if } p_{k}=\stackrel{\circ}{N} & \text { then } & w\left(p_{k}\right)=\stackrel{\circ}{N}_{h}=(h+1) \gamma(\alpha+\beta+\gamma+\delta+h(\alpha+\gamma)(\beta+\delta)) \\
\text { if } p_{k}=\stackrel{\bullet}{N} & \text { then } & w\left(p_{k}\right)=\stackrel{\circ}{N}_{h}=(h+1) \alpha(\alpha+\beta+\gamma+\delta+h(\alpha+\gamma)(\beta+\delta)) \\
\text { if } p_{k}=\stackrel{\circ}{E} & \text { then } & w\left(p_{k}\right)=\stackrel{\circ}{E}_{h}=\beta+\gamma+(h+1)(\alpha \beta+\gamma \delta+2 \beta \gamma) \\
\text { if } p_{k}=\dot{\circ} & \text { then } & w\left(p_{k}\right)=\dot{\circ}_{h}=\alpha+\delta+(h+1)(\alpha \beta+\gamma \delta+2 \alpha \delta) \\
\text { if } p_{k}=\stackrel{\circ}{S} & \text { then } & w\left(p_{k}\right)=\stackrel{\circ}{S}_{h}=\beta \\
\text { if } p_{k}=\stackrel{\bullet}{S} & \text { then } & w\left(p_{k}\right)=\stackrel{\bullet}{S}_{h}=\delta
\end{array}
$$

then equations (22) and (23) hold.

The peculiar choice $q=1-(\alpha+\beta+\gamma+\delta)(\alpha \beta-\gamma \delta) /(\alpha+\delta)(\beta+\gamma)$ also allows a representation like (22), however, the step weights get quite complicated. Fortunately, it is soon noticed that the configuration weights all have a large common factor, and the alert reader will have noticed that this value of $q$ is the same as in Proposition 2 .

In fact, we have the following result.

Proposition 17. Given that $\gamma$ and $\delta$ are positive, $q=1$ and

$$
q=1-(\alpha+\beta+\gamma+\delta)(\alpha \beta-\gamma \delta) /(\alpha+\delta)(\beta+\gamma)
$$

are the only two values of $q$ which allows a representation like (22).

Disregarding the colours totally, we get a slightly simpler representation of the normalisation in the $q=1$ case.

Corollary 18. Let $\tilde{\mathcal{M}}_{n}$ denote the set of (uncoloured) Motzkin paths of length $n$. If the weight of a step $p_{k}$, starting at level $h$, is given by

$$
\begin{array}{lll}
\text { if } p_{k}=N & \text { then } & w\left(p_{k}\right)=\alpha+\beta+\gamma+\delta+h(\alpha+\gamma)(\beta+\delta) \\
\text { if } p_{k}=E & \text { then } & w\left(p_{k}\right)=\alpha+\beta+\gamma+\delta+2(h+1)(\alpha+\gamma)(\beta+\delta) \\
\text { if } p_{k}=S & \text { then } & w\left(p_{k}\right)=(h+1)(\alpha+\gamma)(\beta+\delta)
\end{array}
$$

Then

$$
Z_{n}=\frac{1}{(\alpha \beta-\gamma \delta)^{n}} \sum_{p \in \tilde{\mathcal{M}}_{n}} w(p)
$$

Just as in the $\gamma=\delta=0$ case, we immediately get a generating function for weights.

Corollary 19. Let $f_{w, n}$ be the sum of weights of configurations of length $n$, with exactly $w$ white particles. Further, define $F(t, z)=\sum_{w, n} f_{w, n} t^{w} z^{n}$, and let $\kappa_{h}=z\left(w \stackrel{\circ}{E}_{h}+\dot{E}_{h}\right)$ and 


$$
\begin{aligned}
\lambda_{h}^{\prime}=z^{2}\left(w^{2} \stackrel{\circ}{N}_{h} \stackrel{\circ}{S}+w\left(\stackrel{\circ}{N}_{h} \stackrel{\bullet}{S}+\stackrel{\circ}{N}_{h} \stackrel{\circ}{S}\right)+\stackrel{\circ}{N}_{h} \stackrel{\circ}{S}\right) \text {. Then } \\
F(t, z)=\frac{1}{1-\kappa_{0}-\frac{\lambda_{0}^{\prime}}{1-\kappa_{1}-\frac{\lambda_{1}^{\prime}}{1-\kappa_{2}-\cdots}}} .
\end{aligned}
$$

Remark 1. The normalisation can also be written as the sum over weighted Dyck paths. See Lemma 20 in Section 4.3.

\section{Proofs}

\subsection{Direct proof of Theorem 1}

Let $X=x_{1} \cdots x_{n}$ be any configuration of length $n$. Let $\bar{X}^{k}$ denote the configuration with position $k$ removed, i.e. $\bar{X}^{k}=x_{1} \cdots x_{k-1} x_{k+1} \cdots x_{n}$, and $X^{(k)}$ the configuration with positions $k$ and $k+1$ interchanged, i.e. $X^{(k)}=x_{1} \cdots x_{k-1} x_{k+1} x_{k} x_{k+2} \cdots x_{n}$.

To show that (3) in Theorem 1 defines a stationary distribution, we will show that $f(X)=0$, where

$$
f(X)=\sum_{Y} g_{Y, X} P(Y)
$$

where $P(X)=W(X) / Z_{n}$ (note that $\left.g_{X, X}=-\sum_{Y \neq X} g_{X, Y}\right)$.

Assume that $X$ have the pair $\circ \bullet$ in positions $i_{1}<i_{2}<\cdots<i_{l}$ (position $k$ meaning that $x_{k}=\circ$ and $x_{k+1}=\bullet$ ), and the pair $\bullet \circ$ in positions $j_{1}<j_{2}<\cdots<j_{m}$. Note that $l-1 \leq m \leq l+1$, with $l=m-1$ if and only if $x_{1}=\bullet$ and $x_{n}=\circ$ and $l=m+1$ if and only if $x_{1}=\circ$ and $x_{n}=\bullet$. We have four cases, depending on $x_{1}$ and $x_{n}$. Assume $x_{1}=\circ$ and $x_{n}=\bullet$, the remaining cases being analogous.

Equation (28) then becomes

$$
f(X)=\gamma P\left(\bullet \bar{X}^{1}\right)+\delta P\left(\bar{X}^{n} \circ\right)+\eta \sum_{k=1}^{l} P\left(X^{\left(i_{k}\right)}\right)+q \sum_{k=1}^{m} P\left(X^{\left(j_{k}\right)}\right)-(\beta+\alpha+l q+m \eta) P(X)
$$

Now, by $(2 \mathrm{~b})-(2 \mathrm{~d})$,

$$
\begin{aligned}
& \gamma P\left(\bullet \bar{X}^{1}\right)-\alpha P(X)=-\frac{Z_{n-1}}{Z_{n}} P\left(\bar{X}^{1}\right) \\
& \delta P\left(\bar{X}^{n} \circ\right)-\beta P(X)=-\frac{Z_{n-1}}{Z_{n}} P\left(\bar{X}^{n}\right) \\
& \eta P\left(X^{\left(i_{k}\right)}\right)-q P(X)=\frac{Z_{n-1}}{Z_{n}}\left(P\left(\bar{X}^{i_{k}}\right)+P\left(\bar{X}^{i_{k}+1}\right)\right) \text { and } \\
& q P\left(X^{\left(j_{k}\right)}\right)-\eta P(X)=-\frac{Z_{n-1}}{Z_{n}}\left(P\left(\bar{X}^{j_{k}}\right)+P\left(\bar{X}^{j_{k}+1}\right)\right)
\end{aligned}
$$


Inserting in (29) gives

$$
f(X)=-\frac{Z_{n-1}}{Z_{n}}\left(\hat{P}(1, n)-\sum_{k=1}^{l} \hat{P}\left(i_{k}, i_{k}+1\right)+\sum_{k=1}^{m} \hat{P}\left(j_{k}, j_{k}+1\right)\right)
$$

where $\hat{P}(i, j)=P\left(\bar{X}^{i}\right)+P\left(\bar{X}^{j}\right)$. Finally we note that $\bar{X}^{i_{1}}=\bar{X}^{1}, \bar{X}^{i_{k}+1}=\bar{X}^{j_{k}}, 1 \leq k<l$, $\bar{X}^{i_{k}}=\bar{X}^{j_{k-1}+1}, 1<k \leq l$ and $\bar{X}^{i_{l}+1}=\bar{X}^{n}$. The first assertion is true since the first $\bullet$ in $X$ is in position $i_{1}+1$, so both $x_{1}=0$ and $x_{i_{1}}=0$ and removing them give the same result. The other assertions are showed similarly. Thus $f(X)=0$, and the Theorem is proved.

\subsection{Proof of Proposition 2.}

We may introduce a factor $C$ in the algebra as follows

$$
\begin{aligned}
W(X) & =1 \text { if } X \in \mathcal{B}_{0} \\
\alpha W(\circ X) & =C W(X)+\gamma W(\bullet X) \\
\beta W(X \bullet) & =C W(X)+\delta W(X \circ) \\
\eta W(A \bullet \circ B) & =C(W(A \circ B)+W(A \bullet B))+q W(A \circ \bullet B)
\end{aligned}
$$

as this does not alter the ratio between weights for configurations of equal length. Choose

$$
C=\frac{\alpha \beta-\gamma \delta}{(\alpha+\delta)(\beta+\gamma)}
$$

and let $\eta=1$ and $q=1-(\alpha+\beta+\gamma+\delta) C$. Straightforward calculations now show that $W(X)=\frac{1}{(\alpha+\delta)^{w}(\beta+\gamma)^{n-w}}$, where $w$ is the number of white particles in $X$, satisfies (32a) (32d), and the Proposition follows.

\subsection{Proof of Proposition 5}

A Dyck path may be defined as a Motzkin path without east steps. Let $\mathcal{D}_{2 n}$ be the set of Dyck paths of lenght $2 n$. The weight of a Dyck path is product of the weight of its steps. Let the weight of a north-east (south-east) step from level $h-1$ to $h$ be $N_{h}\left(S_{h}\right)$, and the denote the weight of a Dyck path $p$ by $v(p)$.

We will use the following lemma.

Lemma 20. With the weights

$$
\begin{aligned}
& N_{h}=\left\{\begin{array}{l}
(h+1)(\alpha+\gamma)(\beta+\delta) / 2, \text { if } h \text { is odd, } \\
\alpha+\beta+\gamma+\delta+h(\alpha+\gamma)(\beta+\delta) / 2, \text { if } h \text { is even, }
\end{array}\right. \\
& S_{h}=1,
\end{aligned}
$$

we have

$$
Z_{n}=\frac{1}{(\alpha \beta-\gamma \delta)^{n}} \sum_{p \in \mathcal{D}_{2 n}} v(p)
$$


Proof. Define a surjection $M: \mathcal{D}_{2 n} \rightarrow \mathcal{M}_{n}$ as follows. For $k=1,2, \ldots, n$, if steps $2 k-1$ and $2 k$ in $p \in \mathcal{D}_{2 n}$ are:

* Both north-east, let step $k$ in $M(p)$ be north-east.

* Both south-east, let step $k$ in $M(p)$ be south-east.

* Otherwise, let step $k$ in $M(p)$ be east.

Given the weights in Corollary 18 for Motzkin paths and the above weights for Dyck paths, it is trivial to check that

$$
w(q)=\sum_{p: M(p)=q} v(p)
$$

and the lemma follows.

Next, we will find a recursion for the sum of weights of Dyck paths which start with $d$ north-east steps.

Let $\mathcal{R}_{2 n}^{d}$ be the set of Dyck paths whose first $d$ steps all are north-east. For a path $p \in \mathcal{R}_{2 n}^{d}$, let $w^{d}(p)$ be the weight of the path without the first $d$ steps. Finally, let

$$
Z_{n}^{d}=\sum_{p \in \mathcal{R}_{2 n}^{d}} v^{d}(p)
$$

Note that $Z_{n}^{0}=Z_{n} /(\alpha \beta-\gamma \delta)^{n}$ and that $Z_{n}^{d}=0$ for $d<0$ and $d>n$.

Considering step $d+1$ we get the following recursion

\section{Lemma 21.}

$$
\begin{aligned}
& Z_{n}^{n}=1, \text { for } n=0,1,2, \ldots \\
& Z_{n}^{d}=N_{d+1} Z_{n}^{d+1}+S_{d-1} Z_{n-1}^{d-1} .
\end{aligned}
$$

It is now straightforward to check that the following expression satisfies the above recursion with the weights given in Lemma 20 .

\section{Lemma 22.}

$$
Z_{n}^{d}=\left(\begin{array}{c}
n-d+\lfloor d / 2\rfloor \\
\lfloor d / 2\rfloor
\end{array}\right) \prod_{i=1}^{n-d} \alpha+\beta+\gamma+\delta+(i+\lfloor(d-1) / 2\rfloor)(\alpha+\gamma)(\beta+\delta) .
$$

Let $d=0$, and the proposition follows. 


\subsection{Proof of Theorem 13}

Let

$$
Q(X)=\sum_{p \in \theta^{-1}(X)} w(p)
$$

We will show that $Q(X)$ satisfies the basic weight algebra (2a)-(2d). By definition, (2a) is fulfilled.

Let $X=A B$ be any configuration, and let $p \in \theta^{-1}(X), a \in \theta^{-1}(A)$ and $b \in \theta^{-1}(B)$ be such that $p=a b$.

We have

$$
\begin{aligned}
& w(\stackrel{\circ}{E} p)=\stackrel{\circ}{E}_{0} w(p)=w(p) / \alpha, \text { and } \\
& w(p \stackrel{\bullet}{E})=\stackrel{\bullet}{E}_{0} w(p)=w(p) / \beta
\end{aligned}
$$

Summing over $p \in \theta^{-1}(X)$ gives $(2 \mathrm{~b})$ and $(2 \mathrm{c})$.

Next assume that part $a$ ends at level $h$. Then

$$
\begin{aligned}
& w(a \stackrel{\circ}{E} \dot{E} b)=w(a \dot{E} \stackrel{\circ}{E} b)=w(a b) \stackrel{\circ}{E}_{h} \dot{E}_{h}, \\
& w(a N S b)=w(a b) N_{h} S_{h+1}, w(a S N b)=w(a b) N_{h-1} S_{h}, \\
& w(a \stackrel{\circ}{E} b)=w(a b) \stackrel{\circ}{E}_{h}, \text { and } w(a \dot{E} b)=w(a b) \dot{E}_{h} .
\end{aligned}
$$

One can easily check that

$$
\stackrel{\circ}{E}_{h} \dot{\bullet}_{h}+N_{h} S_{h+1}=\stackrel{\circ}{E}_{h}+\stackrel{\bullet}{E}_{h}+q\left(\stackrel{\circ}{E}_{h} \dot{E}_{h}+N_{h-1} S_{h}\right)
$$

Thus

$$
w(a \stackrel{\circ}{E} \stackrel{\circ}{E} b)+w(a N S b)=w(a \stackrel{\circ}{E} b)+w(a \dot{E} b)+q(w(a \stackrel{\circ}{E} \dot{E} b)+w(a S N b)),
$$

and summing over $p \in \theta^{-1}(X)$ gives $(2 \mathrm{~d})$.

\subsection{Proof of Theorem 16}

We will show that the weights of generalised bicoloured Motzkin paths with weights as given in the Theorem satisfy the algebra in Definition 1. Then, since it is trivial to check that the Theorem holds for $n=1$, the general case follows.

By definition, (2a) is fulfilled.

For a path $p$, we have $w(\stackrel{\circ}{E} p)=\stackrel{\circ}{E}_{0} w(p) /(\alpha \beta-\gamma \delta)$ and $w(\stackrel{\bullet}{E} p)=\dot{\bullet}_{0} w(p) /(\alpha \beta-\gamma \delta)$, so

$$
\alpha w(\stackrel{\circ}{E} p)-\gamma w(\stackrel{\bullet}{E} p)=(\alpha(\beta+\gamma) w(p)-\gamma(\alpha+\delta) w(p) /(\alpha \beta-\gamma \delta)=w(p) .
$$

This is $(2 \mathrm{~b}) ;(2 \mathrm{c})$ is analogous. 
To show (2d), let $p$ be decomposed as $a b$, with part $a$ ending at level $h$. Let $a \circ \bullet b(a \bullet \circ b)$ denote the set of possible bicoloured Motzkin paths starting with $a$, then a white (black) step, then a black (white) step, and ending with $b$.

Then

$$
\begin{aligned}
w(a \bullet \circ) & =w(a b)\left(\stackrel{\bullet}{E}_{h} \stackrel{\circ}{E}_{h}+\stackrel{\circ}{S}_{h} \stackrel{\circ}{N}_{h}+\stackrel{\circ}{N}_{h+1} \stackrel{\circ}{S}_{h+1}\right) /(\alpha \beta-\gamma \delta)^{2}, \\
w(a \circ \bullet b) & =w(a b)\left(\stackrel{\circ}{E}_{h} \dot{\bullet}_{h}+\stackrel{\circ}{S}_{h} \dot{\circ}_{h}+\stackrel{\circ}{N}_{h+1} \dot{\leftrightarrow}_{h+1}\right) /(\alpha \beta-\gamma \delta)^{2}, \\
w(a \stackrel{\bullet}{E} b) & =w(a b) \dot{\bullet}_{h} /(\alpha \beta-\gamma \delta), \text { and } \\
w(a \stackrel{\circ}{E} b) & =w(a b) \stackrel{\circ}{E}_{h} /(\alpha \beta-\gamma \delta) .
\end{aligned}
$$

Basic algebra now shows that

$$
w(a \bullet \circ)-w(a \circ \bullet b)=w(a \stackrel{\circ}{E} b)+w(a \stackrel{\bullet}{E} b),
$$

as required.

\subsection{Proof of Proposition 17.}

Using the algebra, we get, with $S=\alpha+\beta+\gamma+\delta, P=(\alpha+\delta)(\beta+\gamma)$,

$$
\begin{aligned}
W^{\prime}(\circ) & =(\alpha \beta-\gamma \delta) W(\circ)=\beta+\gamma=\stackrel{\circ}{E}_{0} \\
W^{\prime}(\bullet) & =(\alpha \beta-\gamma \delta) W(\bullet)=\alpha+\delta=\stackrel{\bullet}{E}_{0} \\
W^{\prime}(\circ \circ) & =(\alpha \beta-\gamma \delta)(\alpha \beta-q \gamma \delta) W(\circ \circ)=\beta \gamma S+(\beta+q \gamma)(\beta+\gamma)=\stackrel{\circ}{E}_{0} \stackrel{\circ}{E}_{0}+\stackrel{\circ}{N}_{1} \stackrel{\circ}{S}_{0} \\
W^{\prime}(\bullet \bullet) & =(\alpha \beta-\gamma \delta)(\alpha \beta-q \gamma \delta) W(\bullet \bullet)=\alpha \delta S+(\alpha+q \delta)(\alpha+\delta)=\dot{\bullet}_{0} \dot{\bullet}_{0}+\stackrel{\bullet}{N}_{1} \dot{\bullet}_{0} \\
W^{\prime}(\circ \bullet) & =(\alpha \beta-\gamma \delta)(\alpha \beta-q \gamma \delta) W(\circ \bullet)=\gamma \delta S+P=\stackrel{\circ}{E}_{0} \stackrel{\circ}{E}_{0}+\stackrel{\circ}{N}_{1} \stackrel{\bullet}{S}_{0} \\
W^{\prime}(\bullet \circ) & =(\alpha \beta-\gamma \delta)(\alpha \beta-q \gamma \delta) W(\bullet \circ)=\alpha \beta S+q P=\stackrel{\circ}{E}_{0} \stackrel{\circ}{E}_{0}+\stackrel{\circ}{N}_{1} \stackrel{\circ}{S}_{0}
\end{aligned}
$$

Thus, it is required that

$$
\begin{aligned}
\left(W^{\prime}(\circ \circ)-\stackrel{\circ}{E}_{0} \stackrel{\circ}{E}_{0}\right)\left(W^{\prime}(\bullet \bullet)-\dot{E}_{0} \dot{E}_{0}\right) & =\left(W^{\prime}(\circ \bullet)-\stackrel{\circ}{E}_{0} \dot{E}_{0}\right)\left(W^{\prime}(\bullet \circ)-\dot{\bullet}_{0} \stackrel{\circ}{E}_{0}\right) \\
& \Longleftrightarrow \\
(q-1) \gamma \delta(S(\alpha(\beta+\gamma)+\beta(\alpha+\delta))+(q-1) P) & =(q-1) \gamma \delta S P
\end{aligned}
$$

assuming $\gamma>0$ and $\delta>0, q=1$ and $q=1-(\alpha \beta-\gamma \delta) S / P$ are the only two solutions to this equation. 


\section{Marked configurations and the M-PASEP}

In this section we define a larger Markov chain, the M-PASEP, which we use to study the stationary distribution of the original PASEP. In particular we show that the stationary distributions of the M-PASEP and the PASEP are simply related.

\subsection{Marked configurations}

We enlarge the state space of the original chain by adding "marked" configurations (hence the "M" in M-PASEP).

Definition 5. A marked configuration $(X, i, D)$ of size $n$ consists of a basic configuration $X \in \mathcal{B}_{n}$, an integer $i \in[0, n]$ and a "direction" $D \in\{L, R, S, N\}$. The directions are $L$ for "left", $R$ for "right", S for "stable" and $N$ for "nothing". The possible values of D depend on the values of $X$ and $i$. All triples satisfying the following conditions occur:

* for all $X$ and all $i \in[0, n]: D=N$.

* for $X=\circ A$ and $i=0: D \in\{R, S\}$.

* for $X=\bullet A$ and $i=0: D \in\{S\}$.

* for $X=A \circ$ and $i=n: D \in\{S\}$.

* for $X=A \bullet$ and $i=n: D \in\{L, S\}$.

* for $X=A \bullet \circ B,|A| \in[0, n-2]$ and $i=|A|+1: D \in\{L, R, S\}$.

* for $X=A \circ \bullet B,|A| \in[0, n-2]$ and $i=|A|+1: D \in\{S\}$.

We define a projection $U(M)=X$ from a marked configuration $M=(X, i, D)$ to the corresponding unmarked configuration, $X$. We denote the set of all marked configurations of size $n$ by $\mathcal{M}_{n}$.

These are the marked configurations for $n=2$ :

$$
\begin{aligned}
& (\circ \circ, 0, R), \quad(\circ \circ, 0, S), \quad(\circ \circ, 0, N), \quad(\circ \circ, 1, N), \quad(\circ \circ, 2, S), \quad(\circ \circ, 2, N) \\
& (\circ \bullet, 0, R), \quad(\circ \bullet, 0, S), \quad(\circ \bullet, 0, N), \quad(\circ \bullet, 1, S), \quad(\circ \bullet, 1, N), \quad(\circ \bullet, 2, L), \quad(\circ \bullet, 2, S), \quad(\circ \bullet, 2, N), \\
& (\bullet \circ, 0, S), \quad(\bullet \circ, 0, N), \quad(\bullet \circ, 1, L), \quad(\bullet \circ, 1, R), \quad(\bullet \circ, 1, S), \quad(\bullet \circ, 1, N), \quad(\bullet \circ, 2, S), \quad(\bullet \circ, 2, N), \\
& (\bullet \bullet, 0, S), \quad(\bullet \bullet, 0, N), \quad(\bullet \bullet, 1, N), \quad(\bullet \bullet, 2, L), \quad(\bullet \bullet, 2, S), \quad(\bullet \bullet, 2, N)
\end{aligned}
$$




\subsection{The M-PASEP chain}

We define the M-PASEP chain, $C$, whose state space is the union of the basic and marked configurations. For any $X \in \mathcal{B}_{n}$ and $M \in \mathcal{M}_{n}$ the transition probabilities between states in the chain are given by

$$
\begin{aligned}
& \text { if } \quad U(M)=X \text { then } \quad C_{X, M}=\frac{W(M)}{(n+1) W(X)} \\
& \text { if } \quad U(T(M))=X \text { then } C_{M, X}=1 \\
& \text { otherwise } \quad C_{X, M}=C_{M, X}=C_{X, X}=C_{M, M}=0 \text {, }
\end{aligned}
$$

where $W(M)$ is the weight of a marked configuration which we define below and $T: \mathcal{M}_{n} \mapsto$ $\mathcal{M}_{n}$ is a weight preserving bijection given in Definition 7 below.

Definition 6. The weight of a marked configuration $M$ is defined in terms of the basic weights of unmarked configurations as follows:

$$
\begin{aligned}
W(\circ A, 0, R) & =W(A) \\
W(\circ A, 0, S) & =W(\bullet A, 0, S)=\gamma W(\bullet A) \\
W(A \bullet, n, L) & =W(A) \\
W(A \bullet, n, S) & =W(A \circ, n, S)=\delta W(A \circ) \\
W(A \bullet \circ B, i, R) & =W(A \circ B) \quad \text { if } A \in \mathcal{B}_{i-1} \\
W(A \bullet \circ B, i, L) & =W(A \bullet B) \quad \text { if } A \in \mathcal{B}_{i-1} \\
W(A \bullet \circ B, i, S) & =W(A \circ \bullet B, i, S)=q W(A \circ \bullet B) \quad \text { if } \quad A \in \mathcal{B}_{i-1} \\
W(X, i, N) & =W(X)-\sum_{D \neq N} W(X, i, D) .
\end{aligned}
$$

We will refer to these weights as M-basic weights.

Note that the chain alternates between marked and basic configurations. The state graph of the chain $C$ for $n=2$ is shown in Figure 5. The weights of marked and unmarked configurations are simply related and from Definitions 1 and 6 we get:

Lemma 23. For all $X \in \mathcal{B}_{n}$ and $i \in[0, n]$ :

$$
\sum_{D} W(X, i, D)=W(X)
$$

and

$$
\sum_{M: U(M)=X} W(M)=(n+1) W(X) .
$$

The stationary distribution of the PASEP chain is simply related to that of the new chain $C:$ 


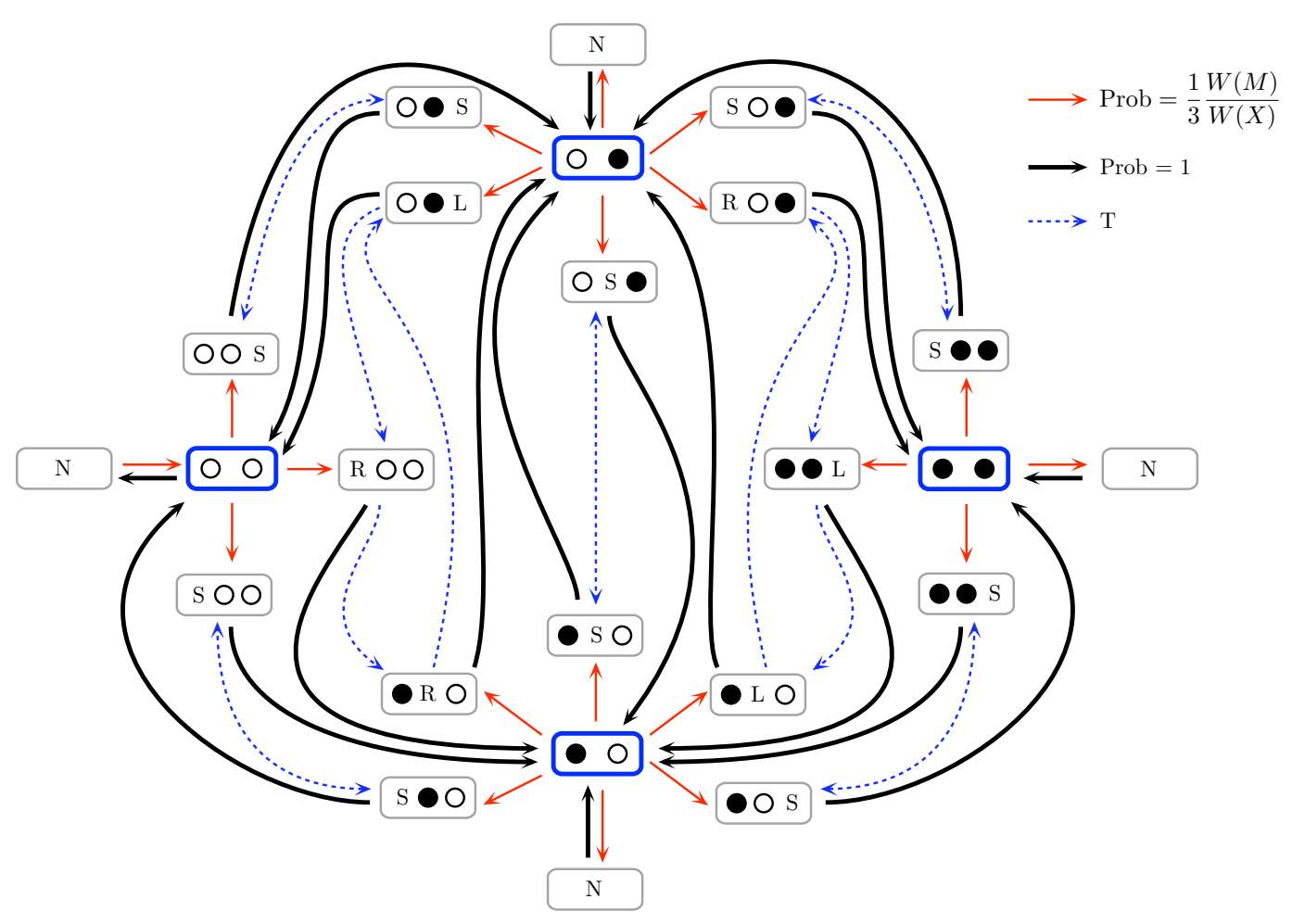

Figure 5: The M-PASEP chain $C$ for $n=2$. Each marked state $(X, i, D)$ is written as the configuration $X$ with its direction $D$ in position $i$. The dashed lines show the action of the bijection $T$.

Proposition 24. The conditional stationary probability of finding the M-PASEP chain, C, in a state $Y$ given that it is in an unmarked state is related to the stationary distribution of $P A S E P$ chain by

$$
\mathrm{P}_{\infty}^{C}\left(Y \text { given that } Y \in \mathcal{B}_{n}\right)=\mathrm{P}_{\infty}(Y)
$$

This proposition relates the stationary distribution of the two chains, but does not tell us what the distributions are. We prove the above proposition in the next section and also expand it to give the proof of Theorem 1.

\section{The stationary distribution for the M-PASEP and proof of Theorem 1}

To prove stationarity we need two major ingredients. The first is a bijection between states on the M-PASEP chain and the second is lemma which gives conditions under which a Markov chain may be altered while leaving its stationary distribution essentially unchanged. 


\subsection{The bijection}

Definition 7. We define a mapping $T: \mathcal{M}_{n} \rightarrow \mathcal{M}_{n}$. Let $M=(X, i, D) \in \mathcal{M}_{n}$. If $D=N$ we define $T(M)=M$. Otherwise we define the mapping by the following algorithm:

$*$ if $i=0$ then the colour of the first particle is changed and

$\diamond$ if $D=S$ then $i$ and $D$ are unchanged, or

$\diamond$ if $D=R$ then "shuffle $M$ right".

$\diamond$ Note that $M=(X, 0, L)$ cannot occur.

* if $i \in[1, n-1]$ then swap the $i^{\text {th }}$ and $i+1^{\text {th }}$ particles and

$\diamond$ if $D=S$ then $i$ and $D$ are unchanged, or

$\diamond$ if $D=L$ then "shuffle $M$ left".

$\diamond$ if $D=R$ then "shuffle $M$ right".

$*$ if $i=n$ then the colour of the last particle is changed and

$\diamond$ if $D=S$ then $i$ and $D$ are unchanged, or

$\diamond$ if $D=L$ then "shuffle $M$ left".

$\diamond$ Note that $M=(X, n, R)$ cannot occur.

where "shuffle $M$ right" means

* choose the minimal $j \in(i, n)$ such that the $j^{\text {th }}$ particle is black and the $(j+1)^{\text {th }}$ particle is white.

* if such a $j$ exists then set $i=j$ and $D=R$

* otherwise set $i=n$ and $D=L$.

and "shuffle $M$ left" means

* choose the maximal $j \in[0, i)$ such that the $j^{\text {th }}$ particle is black and the $(j+1)^{\text {th }}$ particle is white.

* if such a $j$ exists then set $i=j$ and $D=L$

* otherwise set $i=0$ and $D=R$.

Some examples of this mapping are given below

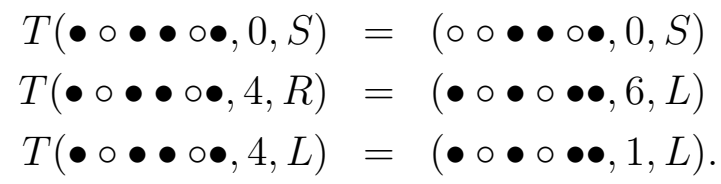


Proposition 25. The mapping $T$ defined in Definition 7 is a bijection from $\mathcal{M}_{n}$ to itself and $\forall M \in \mathcal{M}_{n}$

$$
\text { either } \quad T(M)=M \quad \text { or } \quad T^{2}(M)=M \quad \text { or } \quad T^{n+1}(M)=M .
$$

The mapping is also weight-preserving: $W(T(M))=W(M)$.

Proof. It follows directly from the definition that if $D=N$ then $T(M)=M$ and if $D=S$ then $T^{2}(M)=M$. The weight is invariant in both cases. To show that if $D=L, R$ then $T^{n+1}(M)=M$, we use a one-to-n+1 weight preserving correspondence between the basic configurations in $\mathcal{B}_{n-1}$ and marked configurations in $\mathcal{M}_{n}$ with $D=L, R$.

Start with a configuration $X$ in $\mathcal{B}_{n-1}$. Suppose that the white particles are located at positions $j_{1}<j_{2}<\ldots<j_{i}$ and that the black particles are located at positions $j_{i+2}>$ $j_{i+3}>\ldots>j_{n}$. Now create $n+1$ marked configurations $M_{0}, M_{1}, \ldots, M_{n}$ as follows :

$* M_{0}=(\circ X, 0, R)$.

* $M_{l}=\left(X_{l}, j_{l}, R\right)$ where $X_{l}$ is obtained from $X$ by replacing the $\circ$ located at $j_{l}$ by $\bullet \circ$ with $1 \leq l \leq i$.

$* M_{i+1}=(X \bullet, n, L)$

* $M_{l}=\left(X_{l}, j_{l}, L\right)$ where $X_{l}$ is obtained from $X$ by replacing the $\bullet$ located at $j_{l}$ is replaced by $\bullet$ with $i+2 \leq l \leq n$.

One can check that $T\left(M_{i}\right)=M_{i+1}, 0 \leq i \leq n-1$ and $T\left(M_{n}\right)=M_{0}$. Moreover, the definition of the weight of marked configurations implies that $W\left(M_{i}\right)=W(X)$ for $i=0 \ldots n$ and so the weight is invariant under $T$.

\subsection{The stationary distribution}

In order to show that the stationary state of M-PASEP chain $C$ is simply related to that of the PASEP we use the following lemma:

Lemma 26. Consider a Markov chain $C_{1}$ with a transition from a state a to a state $b$ with probability $r$. We replace the arc $\overrightarrow{a b}$ by the subgraph $\mathcal{H}$ as shown in Figure 6 to create a new chain $C_{2}$. Let $H$ be the set of vertices in $\mathcal{H} \backslash\{a, b\}$. If:

* the weighted sum over all directed spanning trees in $\mathcal{H}$ rooted at $b$ is equal to $r$, and

* the weighted sum over all directed spanning forests of $\mathcal{H}$ that contain 2 components (one rooted at $a$ and one rooted at $b$ ) is equal to 1 ,

then $\operatorname{Pr}_{\infty}^{C_{2}}(x$ given that $x \notin H)=\operatorname{Pr}_{\infty}^{C_{1}}(x)$, where $\operatorname{Pr}_{\infty}^{C_{i}}(x)$ is the stationary state probability of finding chain $C_{i}$ in state $x$. 

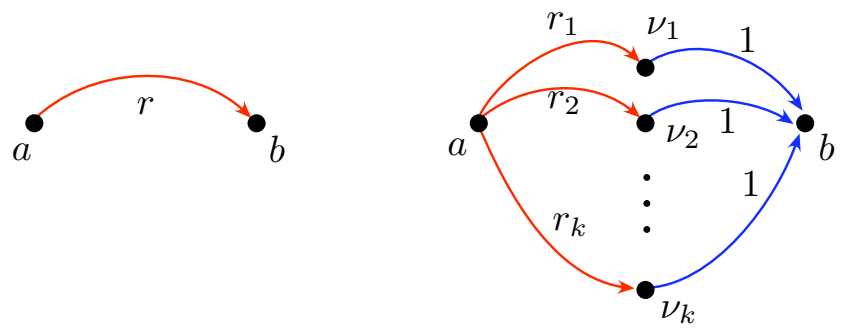

Figure 6: Replace the arc (with transition probability $r$ ) from $a$ to $b$ by a subgraph $\mathcal{H}$ (with $\left.r=r_{1}+\cdots+r_{k}\right)$.

This follows from the Markov-Tree Theorem [5] and can be proved by applying the Matrix-tree theorem to a transition matrix, (it can also be proved combinatorially).

From the definitions of the $M$-basic weights it follows directly that the conditions of Lemma 26 are satisfied (the weights were originally constructed so that the conditions of the lemma are satisfied) and hence Proposition 24 holds. This shows that the two stationary distributions are simply related but does not tell us what they are. The following proposition does this and together with Proposition 24 implies Theorem 1.

Proposition 27. Let $Y$ be a state in the M-PASEP chain $C$, then:

$$
\begin{aligned}
\mathrm{P}_{\infty}^{C}\left(Y \text { given that } Y \in \mathcal{B}_{n}\right) & =\frac{W(Y)}{Z_{n}}, \text { and } \\
\mathrm{P}_{\infty}^{C}\left(M \text { given that } M \in \mathcal{M}_{n}\right) & =\frac{W(Y)}{(n+1) Z_{n}} .
\end{aligned}
$$

where $W(Y)$ is an M-basic weight.

Proof. To prove stationarity we need to show that the above probabilities are unchanged under the action of the transitions. Denote the probability of finding the chain $C$ in state $Y$ at time $t$ by $\operatorname{Pr}(C(t)=Y)$.

Now suppose that

$$
\begin{aligned}
\operatorname{Pr}\left(C(t)=Y \text { given that } Y \in \mathcal{B}_{n}\right) & =\frac{W(Y)}{Z_{n}}, \text { and } \\
\operatorname{Pr}\left(C(t)=M \text { given that } M \in \mathcal{M}_{n}\right) & =\frac{W(M)}{(n+1) Z_{n}}
\end{aligned}
$$


Then because the chain alternates between basic and marked states we have

$$
\begin{aligned}
\operatorname{Pr}\left(C(t+1)=Y \in \mathcal{B}_{n}\right) & =\sum_{M: U(T(M))=Y} \operatorname{Pr}(C(t)=M) \\
& =\sum_{M: U(T(M))=Y} \frac{1}{n+1} \frac{W(M)}{Z_{n}} \\
& =\sum_{M: U(T(M))=Y} \frac{1}{n+1} \frac{W(T(M))}{Z_{n}} \\
& =\sum_{M: U(M)=Y} \frac{1}{n+1} \frac{W(M)}{Z_{n}} \\
& =\frac{W(Y)}{Z_{n}}
\end{aligned}
$$

Above we have used Lemma 23 and the fact that $T$ is a weight preserving bijection (Proposition 25). Similarly we get:

$$
\begin{aligned}
\operatorname{Pr}\left(C(t+1)=Y \in \mathcal{M}_{n}\right) & =\frac{W(M)}{(n+1) W(U(Y))} \operatorname{Pr}(C(t)=U(Y)) \\
& =\frac{W(Y)}{(n+1) W(U(Y))} \frac{W(U(Y))}{Z_{n}}=\frac{W(Y)}{(n+1) Z_{n}}
\end{aligned}
$$

This completes the proof.

\section{References}

[1] P. Flajolet, Combinatorial aspects of continued fractions, Disc. Math. 32 (1980), 125161.

[2] R. A. Blythe, M. R. Evans, F. Colaiori, F. H. L. Essler, Exact solution of a partially asymmetric exclusion model using a deformed oscillator algebra, J. Phys. A: Math. Gen. 33, 2313-2332 (2000). arXiv: cond-mat/9910242.

[3] P. Biane, Permutations suivant le type d'excèdance et le nombre d'inversions et interprètation combinatoire d'une fraction continue de Heine, European J. Combin. 14, no. 4, 277-284 (1993).

[4] R. Brak, J. Essam, Asymmetric exclusion model and weighted lattice paths, J. Phys. A 37 no. 14, 4183-4217 (2004). arXiv: cond-mat/0311153.

[5] S. Chaiken, A combinatorial proof of the all minors matrix tree theorem, SIAM J. Alg. Disc. Meth. 3 (1982), 319-329. 
[6] A. Claesson and T. Mansour, Counting occurences of a pattern of type (1,2) or (2,1) in permutations, Adv in Appl. Math, 29:293-310 (2002).

[7] R. Clarke, E. Steingrimsson, J. Zeng, New Euler-Mahonian statistics on permutations and words, Adv in Appl. Math, 18(3):237-270 (1997).

[8] B. Derrida, E. Domany, D. Mukamel, An exact solution of the one dimensional asymmetric exclusion model with open boundaries J. Stat. Phys. 69, 667-687 (1992).

[9] B. Derrida, M.R. Evans, Exact correlation functions in an asymmetric exclusion model with open boundaries J. Phys. I France 3, 311-322 (1993).

[10] B. Derrida, M.R. Evans, V. Hakim, V. Pasquier, Exact solution of a 1d asymmetric exclusion model using a matrix formulation J. Phys. A26, 1493-1517 (1993).

[11] B. Derrida, C. Enaud, J.L. Lebowitz, The asymmetric exclusion process and Brownian excursions J. Stat. Phys. 115, 365-382 (2004)

[12] E. Duchi and G. Schaeffer, Jumping particles I: maximal flow regime, 16th International Conference on Formal Power Series and Algebraic Combinatorics, (FPSAC'04), Vancouver (Canada).

[13] E. Duchi and G. Schaeffer. A combinatorial approach to jumping particles ii general boundary conditions, Mathematics and computer science, III:399-413, 2006.

[14] E. Duchi and G. Schaeffer. A combinatorial approach to jumping particles, Journal of Combinatorial Theory, Series A., 110(1):1-29, 2005. (arXiv:math.C0/0407519).

[15] G. Schütz and E. Domany Phase transitions in an exactly soluble one-dimensional exclusion process. J. Stat. Phys. 72, 277 - 296 (1993).

[16] M. Uchiyama, T. Sasamoto, M. Wadati, Asymmetric Simple Exclusion Process with Open Boundaries and Askey-Wilson Polynomials, J. Phys. A 37 (2004), no. 18, 49855002. (arXiv: cond-mat/0312457).

[17] G. Viennot, Une théorie combinatoire des polynômes orthogonaux généraux, Notes de Cours, UQAM, 1983. 